\title{
Coding of feature and no-feature events by pigeons performing a delayed conditional discrimination
}

\author{
LOU M. SHERBURNE and THOMAS R. ZENTALL \\ University of Kentucky, Lexington, Kentucky
}

\begin{abstract}
Pigeons' performance of a delayed conditional discrimination with presence versus absence of conditional (sample) stimuli was examined in two experiments. The pigeons showed steeper retention functions with feature (i.e., presence) samples (either food or yellow) than with no-feature (i.e., absence) samples (either no food or no yellow). These results suggest that pigeons code features and respond only by default to test stimuli (comparisons) associated with no features. In contrast, the overall superiority of performance on no-feature-sample trials compared with featuresample trials in both the food/no-food-and yellow/no-yellow-sample tasks was reversed at a 0-sec delay in the food/no-food-sample group, but not in the yellow/no-yellow-sample group. This difference in results with hedonic versus nonhedonic samples suggests that the crossover in delay performance on food/no-food-sample trials is produced by the formation of backward associations between the food-associated comparison stimulus and the food sample.
\end{abstract}

In conditional discriminations that have been used with pigeons, visual cues such as hues or shapes have typically served as samples (e.g., Blough, 1959; Cumming \& Berryman, 1961). More recently, biologically important events such as food and no food have also been found to be highly effective conditional cues, especially in delayed conditional discriminations in which memory plays an important role (see, e.g., Maki, Moe, \& Bierley, 1977; Wilkie, 1978).

The results of research in which food and no-food events have been used as samples in conditional discriminations suggest that performance on no-food-sample trials is generally high, showing a relatively shallow drop as delay increases, compared with performance on food-sample trials (e.g., Colwill, 1984; Grant, 1991; Maki \& Hegvik, 1980; Maki, Olson, \& Rego, 1981; Wilson \& Boakes, 1985). Colwill has interpreted these results as evidence that, although food-sample-trial performance is governed by memory, the appropriate response on a no-food-sample trial is a default response made only in the absence of a food-sample memory (see also Grant, 1991; Maki et al., 1981; Wilson \& Boakes, 1985).

In the studies cited above, "no-food" events generally consisted of the omission of a sample. A problem in the

\footnotetext{
This research was supported by National Science Foundation Grant BSN-8418275 to T. R. Zentall and P. J. Urcuioli and by a grant from the University of Kentucky Research Foundation to T. R. Zentall. The research reported here was based on a thesis submitted by the first author in partial fulfillment of the requirements for the master's degree. We gratefully acknowledge the assistance of Christopher Randall, Karen Roper, and Zhongbiao Zhang in conducting the research. Correspondence concerning this article should be addressed to $\mathbf{L}$. $M$. Sherburne, Department of Psychology, University of Kentucky, Lexington, Kentucky 40506.
}

interpretation of findings of better performance on nostimulus, no-food-sample trials than on food-sample trials is the similarity between delay events and no-food events. It may be that inappropriate "sample coding" occurs on food-sample trials with a delay, because the delay involves the absence of an event (i.e., it is essentially the same as a no-food event) and it always precedes presentations of the comparisons. Furthermore, it may be that the longer it has been since a food-sample presentation, the more likely a pigeon will code the delay as a no-food event.

Grant (1991) has recently reported a similar superiority of performance on no-food-sample trials when a houselight that was otherwise present throughout a session was turned off during the no-food sample. Thus, if the two houselight conditions were sufficiently distinctive, it would appear that the large difference in performance between long-delay, food-sample trials, and no-food-sample trials should not be attributed to confusion between the no-food event and the delay interval.

One purpose of Experiment 1 was to assess foodsample-trial versus no-food-sample-trial delay functions using highly salient no-food events (different from those used by Grant, 1991) that were explicitly distinct from delay events. A second purpose of Experiment 1 was to investigate what appears to be a performance reversal at a 0-sec delay; that is, superior food-sample versus nofood-sample performance. Such an effect has appeared (without analysis or comment) in a number of studies (e.g., Colwill, 1984; Grant, 1991, Figures 1, 2, 3, 4; Maki, 1979, Table 2; Maki \& Hegvik, 1980, Table 2; Wilson \& Boakes, 1985, Figure 1). The presence of a performance "crossover" (superior performance on foodsample trials at a 0-sec delay, but inferior performance on those trials at longer delays) suggests that there may 
be more than one mechanism underlying the differences in delay performance on food-sample versus no-foodsample trials.

\section{EXPERIMENT 1}

Experiment 1 was designed to examine memory for food samples and highly distinctive no-food samples at various retention intervals in a delayed conditional discrimination. In order to make no-food events distinct from delays between samples and comparisons, the no-food events consisted of feeder illumination as well as food presentations that were sufficiently brief to prevent food access. If the similarity between no-food-sample and delay events played a role in the previously reported differences in memory for food versus no-food events, the use of distinctive no-food events as samples should eliminate such differences.

\section{Method}

\section{Subjects}

The subjects were 10 White Carneaux pigeons purchased from the Palmetto Pigeon Plant (Sumter, SC). All the animals had had experience with conditional discrimination tasks involving hue (red and green) and line-orientation samples and line-orientation and shape comparisons. Purina Pigeon Grains, mostly provided during experimental sessions, maintained the pigeons at $80 \%-85 \%$ of their free-feeding weights. The birds were housed in individual wiremesh cages with water and grit freely available. The colony was maintained under a 12:12-h light:dark cycle.

\section{Apparatus \\ The test box was a BRS/LVE (Laurel, MD) Model 132-02 oper- ant chamber; inside measurements were $33 \mathrm{~cm}$ high, $35 \mathrm{~cm}$ from front panel to back wall, and $31 \mathrm{~cm}$ from side to side. The bottom edges of three horizontally aligned rectangular response keys ( $3.0 \mathrm{~cm}$ wide, $2.5 \mathrm{~cm}$ high, and $0.8 \mathrm{~cm}$ apart) were located $21 \mathrm{~cm}$ above the floor. An in-line stimulus projector (Industrial Electronics Engineering, Van Nuys, CA, Model 10 with General Electric No. 1820 lamps) mounted behind each key could illuminate the center key white and the side keys red or green. The red and green hues were produced by Kodak Wratten filters Nos. 26 and 60 , respectively. Mixed-grain presentations were accessible through a horizontally centered $5.2 \times 5.8 \mathrm{~cm}$ aperture, the bottom edge of which was located approximately $8 \mathrm{~cm}$ from the wire-mesh floor. The aperture was illuminated for $2.0 \mathrm{sec}$ whenever the feeder was raised. A shielded houselight (General Electric No. 1820) was mounted $4 \mathrm{~cm}$ from the top of the response panel. Extraneous sounds were masked by white noise at $72 \mathrm{~dB}$ presented through a speaker in the left front wall of the chamber, and by noise from an exhaust fan mounted on the exterior of the chamber. Experimental contin- gencies and data recording were controlled by a microcomputer located in an adjacent room.}

\section{Procedure}

Preliminary training. All of the birds were trained to eat from the feeder and were then shaped to respond to a white center key and to both red and green side keys. A single peck to an illuminated key resulted in $2 \mathrm{sec}$ of reinforcement. Pretraining continued until the birds had responded to 24 center-key presentations and 24 side-key presentations ( 6 presentations of each hue on each side).

Conditional discrimination training. During conditional discrimination training, a single response to the white center key resulted in offset of the keylight and presentation of mixed grain for either $2.0 \mathrm{sec}$ (food sample; F sample) or $0.5 \mathrm{sec}$ (no-food sample; NF sample). Each of the sample presentations was accompanied by $2.0 \mathrm{sec}$ of feeder light. Following offset of the feeder light, red (R) and green $(\mathrm{G})$ comparison stimuli appeared on the side keys. For half of the birds, reinforcement followed a response to the $\mathrm{R}$ comparison following a food sample and to the $G$ comparison following a no-food sample (FR condition). For the remaining birds, the trained associations were reversed (FG condition). A correct response was followed by a 2 -sec presentation of mixed grain and a 10-sec intertrial interval (ITI) with the houselight on. An incorrect response was followed by the illuminated ITI alone. The houselight was off at all other times. The number of each sample type position of the correct comparison, and position of the comparison hues were counterbalanced across trials in each session. Two sequences of trial order were presented in double alternating order over sessions. Each session consisted of 64 trials; sessions were conducted 6 days a week.

Delay phase. After each subject reached criterion (at least $90 \%$ correct on 2 consecutive sessions) and completed a minimum of 12, 64-trial, conditional discrimination training sessions, it began delay training. Mixed delays $(0,1,2$, and $4 \mathrm{sec}$; Delay Set 1$)$ were introduced between samples and comparisons. Each of the delays occurred equally often and delays were counterbalanced over trial type. All stimuli, including the houselight, were off during the delays. When overall performance returned to the criterion level ( 2 consecutive sessions $\geq 90 \%$ correct), or a maximum of 55 sessions had been completed on Delay Set 1 , delay durations were doubled $(0,2,4$, and $8 \mathrm{sec}$; Delay Set 2$)$. The birds were trained on the longer delay set until the criterion performance level was regained, or for a maximum of 45 sessions.

\section{Results}

In order to avoid possible ceiling effects that might reduce or eliminate performance differences between the two trial types, in all analyses only the data from sessions in which overall performance was less than $90 \%$ correct were used. (A mean of 28.6 sessions per bird per delay set was analyzed and a mean of 2.5 sessions per bird per delay set was excluded, due to the performance criterion.) The .05 level of significance was adopted for all analyses.

\section{Acquisition}

The subjects required an average of 11.1 sessions to reach the criterion performance level. Although the birds in the FR condition ( $M=8.4$ sessions) tended to learn more rapidly than those in the FG condition $(M=13.8$ sessions), this difference was not statistically significant $[F(1,8)=4.42]$. A within-subjects analysis of F-sample versus NF-sample-trial performance, pooled over all sessions, suggested that performance was better on NF-sample trials than on F-sample trials, but the effect was not significant $[F(1,9)=4.75]$. In addition, comparison choice latency data from the 3 sessions immediately preceding delay testing were analyzed. Latencies were significantly shorter on NF-sample trials $(M=1.86 \mathrm{sec})$ than on F-sample trials $(M=2.51 \mathrm{sec})[F(1,9)=32.37]$.

\section{Delay Set 1}

When delay data were pooled over sessions on Delay Set 1, performance on NF-sample trials $(M=81.4 \%)$ was significantly better than performance on F-sample trials $(M=71.6 \%)[F(1,8)=37.33]$ There was also a significant delay $\times$ sample interaction $[F(3,24)=49.72]$; 


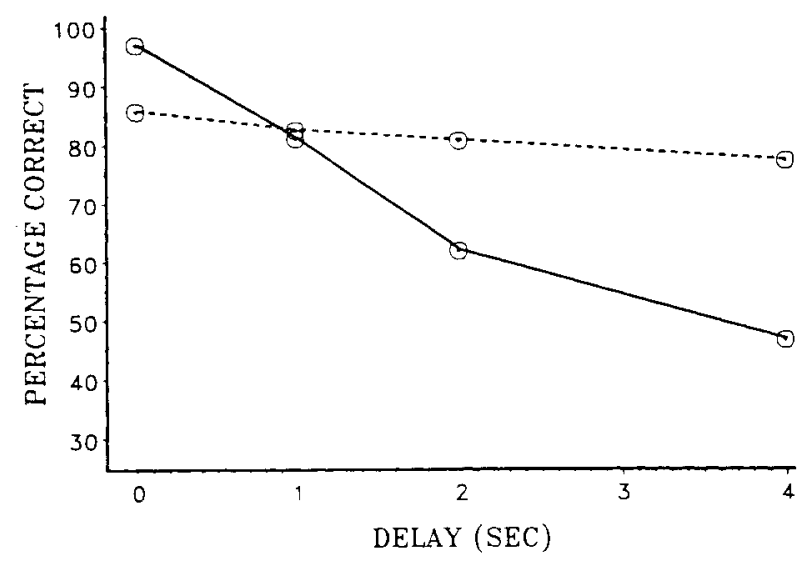

Figure 1. Delay Set 1: Performance on food-sample trials (solid line) and no-food-sample trials (dashed line) as a function of delay in Experiment 1.

the difference in performance between the two sample types increased with increasing length of the delay, as can be seen in Figure 1. NF-sample-trial comparison choice latencies were, again, found to be significantly shorter $(M=1.80 \mathrm{sec})$ than those on F-sample trials $(M=$ $1.96 \mathrm{sec})[F(1,9)=5.84]$.

Simple main effect analyses indicated that performance on NF-sample trials was significantly better than on Fsample trials at both the 2-sec and 4-sec delays $[F(1,9)=$ 26.68 and 92.06 , respectively], but the reverse was true at the 0 -sec delay $[F(1,9)=21.92]$ (means of $96.3 \%$ and $85.9 \%$ correct on trials with $F$ and NF samples, respectively). Performance at the 0 -sec delay represented an increase of $1.5 \%$ correct on F-sample trials (relative to the last two sessions of training) and a decrease of $9.0 \%$ correct on NF-sample trials. It should be noted, however, that the increase in performance on F-sample trials may have been constrained by a performance ceiling ( 5 of the 10 birds performed above $98 \%$ correct on 0 -sec-delay Fsample trials during delay testing).

Although the long-delay superiority of performance on NF-sample trials was present on the first delay session (at the 4-sec delay, 26.2\% correct on F-sample trials vs. $71.2 \%$ correct on NF-sample trials), at the 0 -sec delay there was little difference between F-sample-trial performance $(95.0 \%$ correct) and NF-sample-trial performance ( $92.5 \%$ correct). Instead, the 0-sec-delay trial-type performance crossover appeared to develop with delay training.

Performance on F-sample trials at the longest $(4 \mathrm{sec})$ delay was not significantly different from chance when the data from all sessions were included in the analysis, but a $t$ test performed on the data from only the first delay session revealed that performance was significantly below chance $(M=26.2 \%)$ on F-sample trials at the longest delay $[t(9)=4.67]$. The main effect of delay was also significant $[F(3,24)=102.66]$.

\section{Delay Set 2}

When delays were doubled, overall performance remained higher on NF-sample trials $(M=81.3 \%)$ than on F-sample trials $(M=77.2 \%)[F(1,8)=5.39]$, and the delay $\times$ sample interaction was again significant $[F(3,24)=28.63]$. Although significantly superior performance was found on NF-sample trials at the longest $(8 \mathrm{sec})$ delay $[F(1,9)=30.32]$ (means of $79.7 \%$ and $53.9 \%$ for NF-sample and F-sample trials, respectively), this effect was not accompanied by significantly better F-sample-trial performance at the 0 -sec delay $(F<1)$. For unknown reasons, 1 animal's performance fell to chance levels at all delays for several sessions. After regaining its previous level of performance, this subject exhibited a strong tendency to choose the NF-associated comparison at all delays for several sessions. When this animal's data were excluded from the Delay Set 2 analysis, significantly better F-sample-trial performance was found at the 0-sec delay with this data set as well $[F(1,8)=18.80]$ (means of $97.6 \%$ and $90.1 \%$ for $F$ sample and NF-sample trials, respectively). The main effect of delay was again significant $[F(3,24)=86.84]$. Mean performances with Delay Set 2 (with the deviant bird's data included) are presented in Figure 2.

\section{Discussion}

The first purpose of Experiment 1 was to examine differential memory for food and no-food samples using nofood events that were clearly distinct from delay events. As in previous studies, when the test delays were long, the pigeons showed a strong tendency to respond to the comparison that was associated with the no-food sample. With these distinctive no-food samples, this long-delay "bias" cannot be attributed to the similarity between nofood samples and delay intervals.

One interpretation of these trial-type asymmetry data is that pigeons code both food and no-food samples, but they simply remember no-food samples better than food samples. The finding in the present experiment, that performance on initial long-delay food-sample trials was significantly below chance, is inconsistent with this explanation (see also Colwill, 1984; Grant, 1991; Wilson

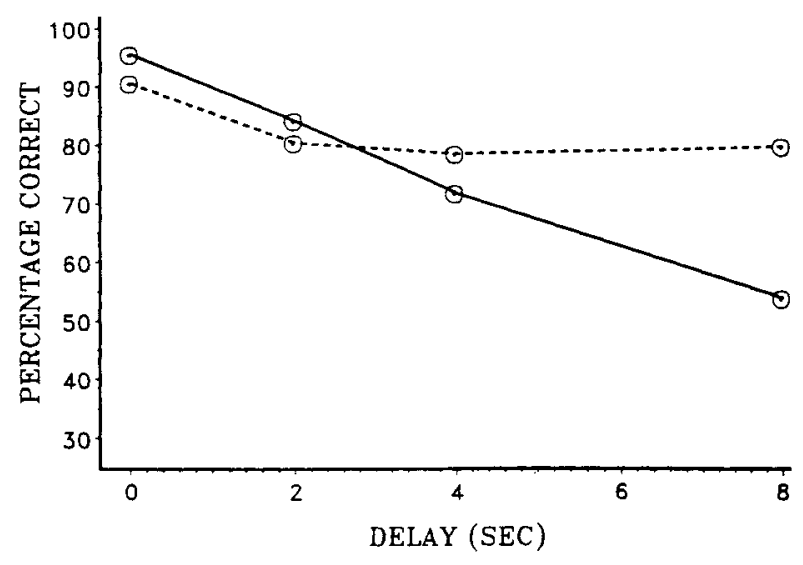

Figure 2. Delay Set 2: Performance on food-sample trials (solid line) and no-food-sample trials (dashed line) as a function of delay in Experiment 1. 
\& Boakes, 1985). Instead, below-chance responding on F-sample trials at long delays suggests that animals select by default the comparison associated with the no-food sample when they cannot remember having been presented the food sample.

The second purpose of Experiment 1 was to assess the reliability of the crossover in food-sample versus no-foodsample-trial performance on 0 -sec-delay trials, which, although unnoted, has appeared in earlier studies (e.g., Colwill, 1984; Grant, 1991, Figures 1, 2, 3, 4; Maki, 1979, Table 2; Maki \& Hegvik, 1980, Table 2; Wilson \& Boakes, 1985, Figure 1). The results of Experiment 1 confirm the finding that 0-sec-delay performance on foodsample trials is significantly better than that on no-foodsample trials.

One explanation for the 0 -sec-delay crossover is that the no-food event was somewhat aversive and resulted in a functional delay (imposed by the pigeon) between nofood samples and comparison choice. Such a delay might result in impaired performance on 0-sec-delay no-foodsample trials relative to that on food-sample trials. However, an analysis of latencies to comparison choice as a function of trial type indicated that the latencies were in fact shorter on no-food-sample trials than on food-sample trials.

An alternative mechanism that could have produced a preference for the food-associated comparison at short delays is the change in the relative density of reinforcement associated with the two comparison stimuli. Although, in our procedure, all correct responses to the comparisons were reinforced, and the long-delay no-food-associated comparison bias produced a high level of performance on no-food-sample trials, this same bias produced a relative reduction in the probability of reinforcement, given a response to the no-food-associated comparison. For example, if, after introduction of delays, a pigeon chooses the correct comparison on $90 \%$ of, say, 100 no-foodsample trials and on $50 \%$ of 100 food-sample trials, it would have responded to the no-food-associated comparison correctly 90 times and incorrectly 50 times. Such a distribution of choices yields a .64 probability correct $[90 /(90+50)]$, given a no-food-associated comparison response. Similarly, the pigeon would have responded to the food-associated comparison correctly 50 times and incorrectly 10 times, yielding a .83 probability correct $[50 /(50+10)]$, given a food-associated comparison response. Thus, all other factors being equal, at short delays, when memory is good and a forgetting-induced nofood-associated comparison bias is absent, this differential probability of reinforcement might result in a small preference for the comparison associated with food. Although it would be expected to be present at all delays, a probability-of-reinforcement effect should be apparent primarily on 0 -sec-delay trials, because on these trials there would be little forgetting of food samples to mask its presence.

A third possible source of a short-delay food-associated comparison bias may be the development of a backward (or bidirectional) association between the correct comparison and the food sample. As pigeons learn the appropriate comparison response to food and no-food samples, they may come to prefer the stimulus that is pecked immediately after a food event over the stimulus that is pecked immediately after a no-food event. The foodassociated comparison may then elicit occasional pecks, resulting in higher performance on food-sample trials than on no-food-sample trials. Such a preference or bias should be particularly apparent at a 0 -sec delay, a point at which little drop in food-sample-trial performance would have occurred.

If the bias to respond to the food comparison at the 0-sec delays in the present study was produced by a backward association, why was no such association evident during training? There was, in fact, a nonsignificant tendency to respond to the comparison associated with no food instead. One possibility is that experience with delays induced the formation of backward associations by encouraging sample "rehearsal." Wagner and Terry (1975) have demonstrated an effect of the rehearsal of unconditional stimulus (US) presentations on backward conditioning in an experiment with rabbits. When the rabbits were exposed to backward pairings involving a "surprising" (and thus rehearsed) US, they displayed stronger backward conditioning than when exposed to backward pairings involving an "expected" US. Although the "surprisingness" of samples was not manipulated in the present experiment, insertion of delays between samples and comparisons may have induced sample rehearsal. If a pigeon is rehearsing the sample representation at the time of comparison presentation, a correct comparison/sample representation association is more likely to occur, and such an association may be the basis of a backward association. The fact that the 0 -sec-delay crossover effect was not present on the first delay session is critical for the probability-of-reinforcement account; it depends on experience with poor performance on food-sample trials and this only occurs at long delays. However, the absence of a 0-sec-delay crossover effect on the first delay session is also compatible with a backward association account, because the rehearsal process may depend on experience with delays.

The backward conditioning account of the 0-sec-delay crossover effect is based on the fact that training involves biologically important samples that result in a comparison bias through backward associations, whereas the probability-of-reinforcement explanation does not require samples with differential hedonic value. In order to differentiate between these interpretations, hedonically neutral samples (i.e., yellow and no yellow) were used in Experiment 2, in addition to the food and no-food samples described earlier. If the tendency to peck comparisons associated with food samples at the 0-sec delay resulted from backward associations between the hue comparisons and the hedonic samples, then no such 0-sec-delay bias should occur with yellow and no-yellow samples. On the other hand, if the 0-sec-delay food- 
associated comparison bias was produced by a probabilityof-reinforcement effect, then sample-type crossover results similar to those found in the first experiment should be found when yellow versus no-yellow samples are used.

\section{EXPERIMENT 2}

The purpose of Experiment 2 was to determine whether the 0 -sec-delay preference for the comparison stimulus associated with the food sample could best be attributed to a difference in the probability of reinforcement associated with responses to the comparisons, or to the formation of a backward association between the food sample and its associated (correct) comparison.

\section{Method}

\section{Subjects}

The subjects were 18 experimentally naive and 4 nonnaive, White Carneaux pigeons purchased from the Palmetto Pigeon Plant (Sumter, SC). The nonnaive birds had had some experience with (but had not performed reliably above $50 \%$ correct on) a dot/no-dotsample 0 -sec-delay conditional discrimination. Purina Pigeon Grains and split peas (provided during experimental sessions) maintained the birds at $80 \%-85 \%$ of their free-feeding weights. The subjects were housed as in Experiment 1.

\section{Apparatus}

The test box was a BRS/LVE operant chamber measuring $32 \mathrm{~cm}$ high, $28 \mathrm{~cm}$ from front panel to back wall, and $35 \mathrm{~cm}$ from side to side. The stimuli were projected on three horizontally aligned circular response keys $(2.5 \mathrm{~cm}$ in diameter and $8.5 \mathrm{~cm}$ between centers of adjacent keys) by in-line stimulus projectors (the same model as that used in Experiment 1) mounted behind each key. The center key could be illuminated white or yellow and the side keys green or red. The yellow, green, and red hues were produced by Kodak Wratten filters Nos. 9, 60, and 26, respectively. Three grain feeders, one containing split peas (used as the food sample), one weighted but empty (used as the no-food sample), and one containing mixed grain (used as the outcome following choice of the correct comparison), were mounted behind the intelligence panel such that if any one of them was raised, it was accessible through a $5.1 \times 5.7 \mathrm{~cm}$ aperture centered on the panel. The bottom edge of the aperture was $7 \mathrm{~cm}$ above the wire-mesh floor and was illuminated whenever any of the feeders was raised. A houselight (General Electric No. 1820) was mounted $4 \mathrm{~cm}$ above the response keys. Extraneous sounds were masked by white noise at $72 \mathrm{~dB}$ presented through a speaker located on the left front wall of the chamber, and by the sound of an exhaust fan mounted on the exterior of the chamber. Experimental contingencies and data recording were controlled by a microcomputer located in an adjacent room.

\section{Procedure}

The experimentally naive birds were randomly assigned to two groups: 8 to the food/no-food-samples (F/NF) group and 10 to the yellow/no-yellow-samples (Y/NY) group. The 4 remaining birds were assigned to the $\mathrm{Y} / \mathrm{NY}$ group.

Preliminary training. The birds were trained to eat from the mixed-grain feeder and then were shaped to peck the white center key and the red and green side keys. A single peck to an illuminated key resulted in $2 \mathrm{sec}$ of reinforcement (mixed grain). Pretraining continued until the birds had responded to 24 center-key presentations and 24 side-key presentations.

Conditional discrimination training. The subjects initiated a trial with a single response to the presentation of the white center key. For F/NF subjects, the response resulted in either an F sample (2-sec presentation of split peas) or an NF sample (2-sec presentation of the empty hopper). Peas were used as the $\mathrm{F}$ sample to provide an F-sample event that was distinct from the outcome of a correct comparison response (i.e., mixed grain). For $\mathrm{Y} / \mathrm{NY}$ subjects, a peck to the white key resulted in a 6-sec presentation of either yellow on the center key accompanied by houselight illumination or $6 \mathrm{sec}$ of houselight illumination only. Following sample offset, $R$ and $G$ comparison stimuli appeared on the side keys. For 4 of the F/NF birds and 6 of the Y/NY birds, reinforcement was given for a response to $\mathrm{R}$ following a feature sample ( $\mathrm{F}$ or $\mathrm{Y}$ ) and for a response to $\mathrm{G}$ following a no-feature sample (NF or NY). For the remaining birds, the trained associations were reversed (feature with $G$, no feature with $\mathrm{R}$ ). Reinforcement of a correct response consisted of a 1.5-sec presentation of mixed grain and a 10-sec ITI with the houselight on. Incorrect responses were followed by the ITI (with houselight) alone. Sample type, position of the correct comparison, and position of the comparison hues were counterbalanced across the 64 trials in each session. Two different sequences of trial order were used.

Delay phase. After the subjects had achieved a criterion of at least $90 \%$ correct on two consecutive sessions and had completed a minimum of 12 sessions, they began delay testing. Mixed delays $(0,1,2$, and $4 \mathrm{sec})$ were introduced between sample offset and comparison onset. All stimuli (including the houselight) were off during delays. Each of the delays occurred equally often and were counterbalanced over trial types. Delay durations were doubled $(0$, 2,4 , and $8 \mathrm{sec}$ ) for each animal after it reached a performance criterion of at least $90 \%$ correct on 2 consecutive days, or completed 10 delay sessions during which performance was $60 \%$ correct or better, whichever came first. Testing with the longer delay set continued until one of the performance criteria was again met.

\section{Results}

In all analyses, only the data for which performance was below $90 \%$ correct were used. Unless otherwise specified, analyses of variance (ANOVAs) were performed on the data from the first 10 sessions at a given delay set. Because the performance of the 4 experienced birds was indistinguishable from that of the other birds, their data were included in the analyses.

\section{Acquisition}

The pigeons achieved the criterion performance level ( $\geq 90 \%$ correct on 2 consecutive sessions) faster on the Y/NY task (14.1 sessions) than on the F/NF task (17.9 sessions); however, the difference was not statistically significant $(F<1)$. Differences in mean sessions to acquisition criterion between the feature- $R /$ no-feature-G conditions and the feature- $\mathrm{G} /$ no-feature- $\mathrm{R}$ conditions (18.5 sessions vs. 17.5 sessions, respectively, for the F/NF task and 12.4 sessions vs. 15.1 sessions, respectively, for the $\mathrm{Y} / \mathrm{NY}$ task) were also not significant (both $F \mathrm{~s}<1$ ). Furthermore, performance pooled over all analyzed sessions did not differ between NF-sample trials and F-sample trials or between $\mathrm{Y}$-sample trials and $\mathrm{NY}$-sample trials (both $F \mathrm{~s}<1$ ).

\section{F/NF: Delay Set 1}

When the data were pooled over sessions on Delay Set 1 , overall performance on NF-sample trials $(M=$ $\mathbf{7 4 . 8 \%}$ correct) was significantly better than performance on F-sample trials $(M=56.3 \%$ correct $)[F(1,6)=$ 
30.04]. There was also a significant delay $\times$ sample interaction $[F(3,18)=38.95]$. The difference between NFsample and $\mathrm{F}$-sample performance increased with length of the delay. As can be seen from the means from Delay Set 1 that are presented in Figure 3, performance on NFsample trials was better than performance on F-sample trials at the long delays $[F(1,7)=39.19,43.16$, and 23.69 at 1,2 , and $4 \mathrm{sec}$, respectively], but the reverse was true at the 0 -sec delay $[F(1,7)=27.26]$ (means of $89.7 \%$ correct and $76.4 \%$ correct for F-sample and NFsample trials, respectively). Once again, although the long-delay, NF-sample-trial performance superiority showed up on the first delay session $(26.6 \%$ correct on F-sample trials vs. $73.5 \%$ correct on NF-sample trials at 4-sec delays), the trial-type performance crossover at the 0 -sec delays was not present on the first delay session ( $82.8 \%$ correct on F-sample trials vs. $84.4 \%$ correct on NF-sample trials). In addition, a $t$ test revealed belowchance, Session 1 responding on F-sample trials at the 4 -sec delay $[t(7)=3.91](M=26.6 \%$ correct $)$. The main effect of delay was also significant $[F(1,7)=66.75]$.

\section{F/NF: Delay Set 2}

When delays were doubled, overall performance was no longer significantly higher on NF-sample trials $(M=$ $73.7 \%$ correct) than on F-sample trials $(M=67.3 \%$ correct) $[F(1,7)=4.15]$, but the delay $\times$ sample interaction was again significant $[F(3,18)=13.77]$. Again, performance was significantly better on NF-sample trials than on F-sample trials at the long delays $[F(1,7)=6.46$ and 19.08 at 4 and $8 \mathrm{sec}$, respectively]. On the other hand, performance was better on F-sample trials $(M=97.0 \%$ correct) than on NF-sample trials $(M=81.4 \%$ correct) at the 0 -sec delay $[F(1,7)=37.39]$. The main effect of delay was again significant $[F(3,18)=45.69]$.

\section{Y/NY: Delay Set 1}

Overall performance was significantly better across delays on NY-sample trials $(M=77.7 \%$ correct $)$ than on

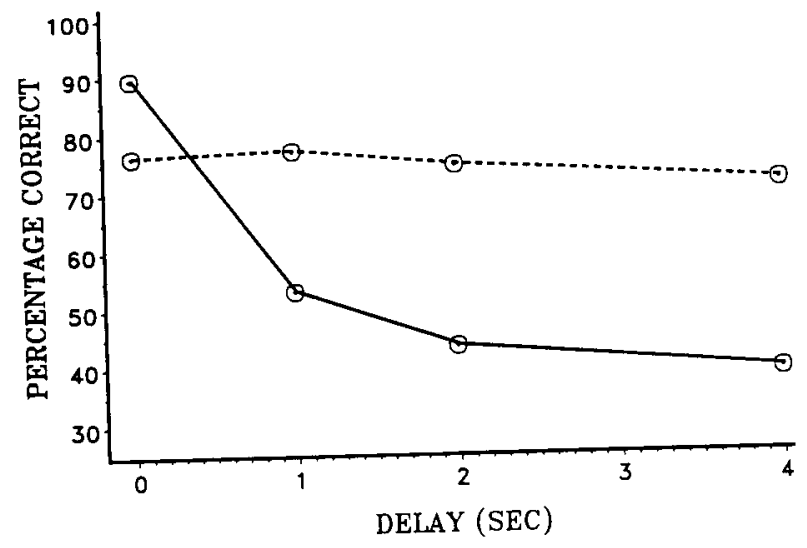

Figure 3. Delay Set 1: Performance on food-sample trials (solid line) and no-food-sample trials (dashed line) as a function of delay in Experiment 2.

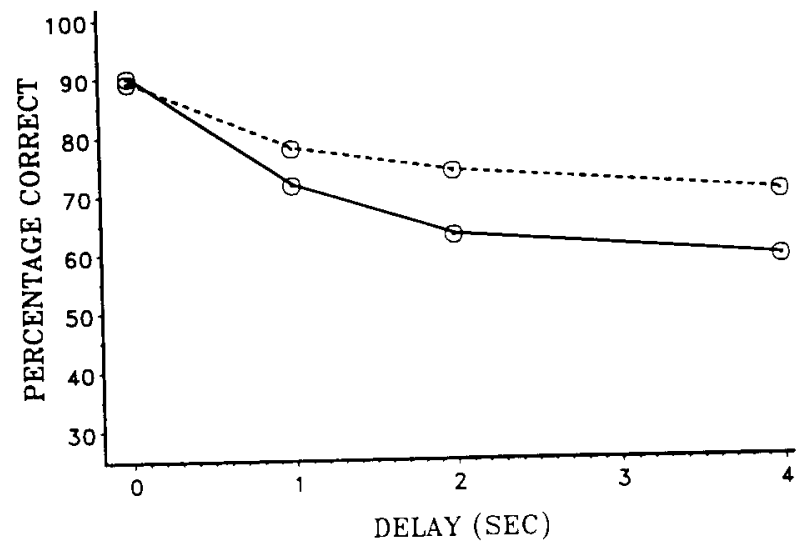

Figure 4. Delay Set 1: Performance on yellow-sample trials (solid line) and no-yellow-sample trials (dashed line) as a function of delay in Experiment 2.

Y-sample trials $(M=70.9 \%$ correct) $[F(1,11)=8.78]$, and there was also a significant delay $\times$ sample interaction $[F(3,33)=3.01]$. As can be seen in Figure 4 , the difference between NY-sample-trial and Y-sample-trial performance increased as a function of delay length. As with the F/NF group, significantly better performance was found on no-feature-sample than on feature-sample (NYsample vs. Y-sample) trials at the long delays ( 2 and $4 \mathrm{sec}$ ) $[F(1,12)=8.32$ and 6.63 , respectively $]$. However, unlike the $F / N F$ group, no significant trial-type effect was found at the 0 -sec delay (means of $90.2 \%$ correct and 89.3\% correct on Y-sample and NY-sample trials, respectively; $F<1)$. Again, the main effect of delay was significant $[F(3,33)=62.85]$.

Although performance on $Y$-sample trials at the 4-sec delay was better than chance when all sessions were included in the analysis, performance on these trials was significantly below chance on the first delay session $[t(12)=2.86](M=39.4 \%$ correct $)$.

\section{Y/NY: Delay Set 2}

When delays were doubled, only the effect of delay remained significant $[F(3,30)=43.32]$.

\section{Between-Groups Comparisons}

Although similar effects were obtained in the two groups with Delay Set 1, effects were larger and more durable in the F/NF group. An ANOVA performed on the Delay Set 1 data from all the birds revealed significantly better overall performance in the $\mathrm{Y} / \mathrm{NY}$ group $(M=74.3 \%$ correct) than in the $\mathrm{F} / \mathrm{NF}$ group $(M=$ $65.6 \%$ correct $),[F(1,147)=22.21]$. In addition, nofeature performance ( $M=76.6 \%$ correct) was better overall than feature performance $(M=65.3 \%$ correct $)$ $[F(1,19)=32.36]$, and feature type interacted with group $[F(1,19)=32.36]$, delay $[F(3,57)=24.52]$, and group $x$ delay $[F(3,57)=11.16]$. The increase in the difference between feature and no-feature performance as a function of delay was larger in the F/NF group than in 
the Y/NY group. The effect of delay was also significant $[F(3,57)=122.42]$. At the 0 -sec delay, the group (Y/NY vs. F/NF) $\times$ sample-type (feature vs. no feature) interaction was also significant $[F(1,19)=9.89]$. On 0 -sec delay trials, the pigeons in the F/NF group preferred the comparison associated with the feature (food) sample over the one associated with the no-feature (no food) sample, whereas no such preference for the comparison associated with the feature (yellow) sample over the no-feature (no yellow) sample was found in the Y/NY group.

\section{Discussion}

The long-delay effect found in Experiment 1-a bias to choose the no-food comparison-was replicated in Experiment 2 with food and empty-hopper samples of equal duration. In the yellow/no-yellow (hue feature) group, a similar delay-performance difference between trial types was seen (i.e., higher accuracy on no-yellow-sample trials than on yellow-sample trials), consistent with recent findings reported by Grant (1991). As in the food-feature group, the birds performed significantly below chance on trials with the long-delay feature (yellow) sample-an indication that the pigeons coded the feature and responded to the comparison associated with the no-feature sample when they had no memory for the feature.

In addition to the long-delay effects, the 0 -sec-delay performance difference found in Experiment 1-higher accuracy on food-sample trials than on no-food-sample trials-was replicated in the food-feature group. On the other hand, no evidence for such a 0 -sec-delay difference in performance between the two trial types was found in the hue-feature group. These results suggest that the higher level of performance on 0-sec-delay food-sample trials as compared with no-food-sample trials was probably not due to relative differences between the two comparisons in the probability of reinforcement given a response. Although the requisite long-delay difference between performances on the two trial types was found in the yellow/no-yellow group, it did not result in a 0 sec-delay crossover as it did in the food/no-food group. Rather, 0-sec-delay performance superiority on foodsample trials appears to suggest the development of backward associations between the comparison and the food sample in the food/no-food group, because, according to this view, only with hedonic samples would one expect to find such a 0 -sec-delay food-associated comparison bias.

It is possible, however, that a difference between yellow-sample-trial and no-yellow-sample-trial performance at the 0 -sec delay did not emerge because of the higher overall level of performance in the hue-feature group (see Figure 4). Higher overall performance means that the difference in performance on yellow-sample versus no-yellow-sample trials is likely to be constrained by a performance ceiling. Because a fairly large difference in performance between the two sample-type trials may be necessary to produce a detectable difference in probability correct given a response (upon which the purported effect is based), these data may not provide a fair test of the hypothesis. In fact, the overall difference between yellow-sample-trial and no-yellow-sample-trial performance was somewhat smaller than the food versus no-food difference. Consequently, the difference between the probability correct given a response to the comparison associated with the yellow sample $(76.1 \%)$ and the probability correct given a response to the no-yellowsample associated comparison $(72.8 \%)$ was only $3.3 \%$. The comparable difference for the food/no-food-sample task was almost twice that $(6.0 \%)$.

The yellow/no-yellow birds were trained and tested with longer $(6 \mathrm{sec})$ samples than were the food/no-food birds $(2 \mathrm{sec})$, on the assumption that food was more salient and perhaps more memorable than a comparable duration of hue stimulus. Apparently, extending the duration of yellow and no-yellow samples resulted in better performance on that task than on the food/no-food-sample task.

In order to get a better estimate of relative delay performance with hedonic versus nonhedonic samples, shortly after completing the test sessions in Experiment 2, 4 of the food/no-food birds were retrained (to the same criterion as in Experiment 2) with 2-sec yellow and no-yellow samples. They were then tested with delays.

As can be seen in Figure 5, performance on yellow/ no-yellow-sample trials by these pigeons was more comparable to performance on food/no-food-sample trials found in Experiment 2 (see Figure 3). Furthermore, the differences between the probability of reinforcement given a response to the feature-associated comparison and the probability of reinforcement given a response to the nofeature-associated comparison were comparable on the two tasks (i.e., $6.0 \%$ for the 2 -sec food condition and $7.0 \%$ for the $2-\mathrm{sec}$ yellow condition).

Once again, however, no evidence of a 0 -sec-delay crossover was found on the yellow/no-yellow task. Mean performance on 0-sec-delay yellow-sample trials was quite similar to that on no-yellow-sample trials $(85.6 \%$ vs. $84.1 \%$ correct on yellow-sample and no-yellow-sample trials, respectively, in contrast to the earlier performances

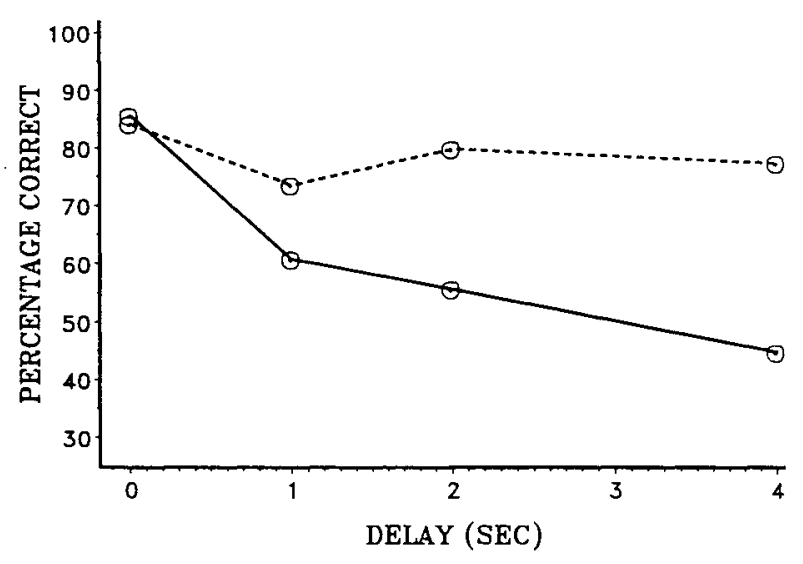

Figure 5. Performance on 2-sec yellow-sample trials (solid line) and 2-sec no-yellow-sample trials (dashed line) as a function of delay in Experiment 2. 
of the same 4 birds on food-sample and no-food-sample trials- $87.2 \%$ vs. $72.5 \%$ correct, respectively). Thus, it appears unlikely that the 0 -sec-delay food/no-food effect found in both Experiments 1 and 2 is attributable to a bias to respond to the comparison associated with the higher probability of reinforcement given a response. Instead, these data are consistent with the development of a backward association between the hue comparison and the food (or no-food) sample. Apparently, this backward association resulted in a bias to respond to the comparison associated with a food sample.

\section{GENERAL DISCUSSION}

The birds trained with food/no-food samples, but not those trained with yellow/no-yellow samples, showed a reversal of the no-feature-associated comparison bias on 0 -sec-delay trials; better performance on feature-sample trials than on no-feature-sample trials. These results cannot be explained in terms of a probability-of-reinforcement effect (i.e., a higher density of reinforcement associated with responses to one of the comparisons than with responses to the other comparison). Instead, these results were interpreted as evidence for the development of a backward association between the food sample and its associated correct comparison.

Although support for the existence of backward excitatory conditioning has generally come from experiments in which Pavlovian procedures were used (e.g., US-CS presentations led to faster acquisition of CS-US associations, as in Hearst, 1989), some evidence suggests that pigeons may form backward associations between stimuli paired in conditional discriminations. Zentall, Sherburne, and Steirn (1992) trained pigeons on a symbolic matching task with differential outcomes for correct comparison choice (e.g., correct responses to the red comparison following a circle sample resulted in the presentation of peas, whereas correct responses to the green comparison following a dot sample resulted in no food, but advancement to the next trial). When in transfer, the food and no-food outcomes replaced the circle and dot samples; the pigeons' comparison choices were predictable from the purported backward associations between outcomes and comparisons established during training.

Consistent with the results of the present experiment, Zentall et al. (1992) suggested that previous attempts to demonstrate the formation of backward associations during conditional discrimination training may have been less successful (e.g., see Hogan \& Zentall, 1977) because all the stimuli trained as associates were nonhedonic.

As mentioned earlier, although the superiority of food-sample-trial performance at 0-sec delay has not been discussed in studies in which samples of food and no food were used, an inspection of the graphs and tables presented in many of these studies suggests an (unanalyzed) 0-secdelay crossover in the context of an food-sample versus no-food-sample-trial long-delay performance difference.

Of particular interest with respect to the present research are Grant's (1991) findings. First, not only did Grant find better delay performance on absence-sample trials than on presence-sample trials with both hedonic and nonhedonic stimuli, but an evaluation of Grant's Figure 1 indicates that there was a 0-sec-delay crossover in the group with hedonic samples, but not in the groups with nonhedonic (color or shape) samples.

Second, when the number of samples associated with each of the comparisons was increased to two (e.g., food-red, horizontal-red, no food-green, vertical-green), the long-delay bias was not observed (Grant, 1991, Experiment 2). Presumably, elimination of the long-delay bias occurred because responding to the green comparison could no longer be under the control of a default rule (e.g., if no memory for food, respond to green). This many-to-one mapping task also led to what appears to be a smaller 0 -sec-delay performance difference than that for one-to-one, sample-to-comparison mapping controls (see Grant, 1991, Figures 2 and 3).

Third, the birds trained to associate a food sample with one comparison and to associate a no-food sample and two different shape samples with the other comparison also showed no long-delay bias, but they did appear to show food-sample-trial performance that was superior to no-food-sample-trial performance at the 0-sec delay. In contrast, the birds trained to associate the no-food sample with one comparison and to associate a food sample and two different shape samples with the other comparison showed the long-delay bias, but showed no 0-sec-delay crossover (see Grant, 1991, Figure 4).

Thus, Grant's (1991, Experiment 3) findings, too, appear to be inconsistent with a probability-of-reinforcement interpretation of the 0 -sec-delay crossover. If the 0 -secdelay effect arises as a result of the differential probability of reinforcement, given a response associated with one of the comparisons, then the 0 -sec-delay effect should depend on the presence of an opposite long-delay effect.

On the other hand, if one assumes that the development of a backward association between the comparison stimulus and a food sample is diminished by the formation of additional associations between that comparison and nonhedonic samples, then Grant's (1991) results appear to be consistent with a backward association interpretation of the 0 -sec-delay effect. First, with a two-to-one, sample-to-comparison mapping (Grant, 1991, Experiment 2), one would expect reduced food-sample-trial performance superiority at the 0 -sec delay, and that is what appears in Grant's Figures 2 and 3. Second, with a threeto-one, sample-to-comparison mapping involving the food sample (Grant, 1991, Experiment 3), one would expect little evidence of better food-sample-trial performance at the 0-sec delay; no crossover appears in Grant's Figure 4. However, in the same figure, with three-to-one, sampleto-comparison mapping involving the no-food sample, food-sample-trial performance appears to be superior to no-food-sample-trial performance at the 0-sec delay. Thus, Grant's results offer additional support for the backward association hypothesis proposed to explain the 0 -sec-delay crossover effect found in the present experiments. 


\section{REFERENCES}

BLough, D. S. (1959). Delayed matching in the pigeon. Journal of the Experimental Analysis of Behavior, 2, 151-160.

Colwill, R. M. (1984). Disruption of short-term memory for reinforcement by ambient illumination. Quarterly Journal of Experimental Psychology, 36B, 235-258.

Cumming, W. W., \& Berryman, R. (1961). Some data on matching behavior in the pigeon. Journal of the Experimental Analysis of Behavior, 4, 281-284.

GRANT, D. S. (1991). Symmetrical and asymmetrical coding of food and no-food samples in delayed matching in pigeons. Journal of Experimental Psychology: Animal Behavior Processes, 17, 186-193.

HeARST, E. (1989). Backward associations: Differential learning about stimuli that follow the presence versus the absence of food in pigeons. Animal Learning \& Behavior, 17, 280-290.

HoGAN, D. E., \& ZENTALL, T. R. (1977). Backward associations in the pigeon. American Journal of Psychology, 90, 3-15.

MAKI, W. S. (1979). Pigeons' short-term memories for surprising vs. expected reinforcement and nonreinforcement. Animal Learning \& Behavior, 7, 31-37.

MAKI, W. S., \& Hegvik, D. K. (1980). Directed forgetting in pigeons. Animal Learning \& Behavior, 8, 567-574.
Maki, W. S., Moe, J. C., \& Bierley, C. M. (1977). Short-term memory for stimuli, responses, and reinforcers. Journal of Experimental Psychology: Animal Behavior Processes, 3, 156-177.

MAKI, W. S., OLSON, D., \& REGo, S. (1981). Directed forgetting in pigeons: Analysis of cue functions. Animal Learning \& Behavior, 9 , 189-195.

WAGNER, A. R., \& TERRY, W. S. (1975). Backward conditioning to a CS following an expected vs. a surprising UCS. Animal Learning \& Behavior, 3, 370-374.

WILKIE, D. M. (1978). Delayed symbolic matching to sample in the pigeon. The Psychological Record, 28, 463-469.

WILSON, B., BoAKES, R. A. (1985). A comparison of the short-term memory performances of pigeons and jackdaws. Animal Leaming \& Behavior, 13, 285-290.

Zentall, T. R., Sherburne L. M., \& Steirn, J. N. (1992). Development of excitatory backward associations during the establishment of forward associations in a delayed conditional discrimination by pigeons. Animal Learning \& Behavior, 20, 199-206.

(Manuscript received February 5, 1992; revision accepted for publication July 29, 1992.) 